\title{
A NOTE ON TWO SET-TRANSITIVE PERMUTATION GROUPS
}

\section{CHONG-YUN CHAO}

In a generalization of permutation groups, Beaumont and Peterson in [1] stated the following definitions: A permutation group $G$ on $n$ letters denoted by $\Omega_{n}=\{1,2, \cdots, n\}$ is said to be $s$ set-transitive $(1 \leqq s \leqq n-1)$ if for every pair of subsets $S$ and $T$ of $\Omega_{n}$, each containing $s$ elements, there exists a permutation in $G$ which carries $S$ into $T$. Also, $G$ is said to be set-transitive if $G$ is $s$ set-transitive for all $s(1 \leqq s \leqq n-1)$. They showed that the set-transitive permutation groups on $n$ letters, other than the symmetric group $S_{n}$ and the alternating group $A_{n}$, are of degree 5,6 and 9. All these groups are doubly transitive except $A_{3}$. They also showed that if $G$ is $s$ set-transitive for at least one $s>1$ then $G$ is primitive. It seems natural to ask whether there are $s$ set-transitive permutation groups which are not doubly transitive for some $s(1<s<n-1)$ besides $A_{3}$ and the 2 set-transitive group on $\Omega_{7}$ generated by (1234567) and (235)(476) and its conjugates in $S_{7}$ (see p. 36 in [1]). It is known that, on p. 94 in [2], a primitive group of degree $n=2 p$ ( $p$ prime) is doubly transitive if $n$ is not of the form $a^{2}+1$. Also, it is not known if $p \neq 5$ there are primitive groups of degree $2 p$ which are not doubly transitive. Hence, the answer to our question depends on $n$. The purpose of this note is, by using elementary concept of graphs and number theory, to present a constructive proof to the following

THEOREm. Let $p$ be a prime such that $p \equiv 3 \bmod 4$. Then there exists an 2 set-transitive permutation group on $\Omega_{p}$ which is not doubly transitive.

The graphs which we consider here are finite, directed and loopless, i.e., by a graph $X$ we mean a finite set $V(X)$, called the vertices of $X$, together with a set $E(X)$, called the edges of $X$, consisting of ordered pairs $[a, b]$ of distinct elements $a, b \in V(X)$. We also assume that there is at most one directed edge between two ordered vertices. The graph with all possible edges is called a complete graph, and the graph with no edges is said to be a null graph. An automorphism $\sigma$ of $X$ is an one-to-one map of $V(X)$ onto $V(X)$ such that $[a \sigma, b \sigma] \in E(X)$ if and only if $[a, b] \in E(X)$. The set of automorphisms of $X$ constitutes a group, denoted by $G(X)$, where the multiplication is the multiplication of permutations. Let $H$ be an abstract finite group and $K$ be a

Received by the editors December 23, 1965. 
subset of $H$. The Cayley graph of $H$ with respect to $K$ ( $K$ does not contain the identity of $H)$ is $X_{H, K}$ with $V\left(X_{H, K}\right)=H$ and $E\left(X_{H, K}\right)$ $=\{[h, h k] ; h \in H, k \in K\}$. If $K$ is the empty set $\varnothing$, then $E\left(X_{H, K}\right)$ is meant to be $\varnothing$, i.e., $X_{H, K}$ is a null graph. Clearly, we have

LEMMA 1. The left regular representations of $H$ is contained in $G\left(X_{H, K}\right)$ for any subset $K$ in $H$.

Lemma 2. Let $X$ be a graph of $n \geqq 3$ vertices. Then $G(X)$ is doubly transitive if and only if $X$ is either the null graph or the complete graph.

Proof. Both the null graph and the complete graph have the symmetric group, $S_{n}$, as their group of automorphisms. Conversely, if $X$ is not the null graph, then there is an edge $[a, b] \in E(X)$. By double transitivity of $G(X)$, all edges belong to $E(X)$, i.e., $X$ is a complete graph.

Now the proof of the theorem goes as follows: Let $H=\left\{e=x^{p}, x^{1}\right.$, $\left.x^{2}, \cdots, x^{p-1}\right\}$ be the cyclic group of order $p$ generated by $x$. We know that the group of automorphisms of $H$, denoted by $A(H)$, is a cyclic group of order $p-1$ on $p$ letters leaving $e$ fixed. Let $\tau$ be a generator of $A(H)$ and let $\sigma=\tau^{2}$, then $\sigma$ is of order $(p-1) / 2$. Let $K=\left\{x \sigma, x \sigma^{2}, \cdots, x \sigma^{(p-1) / 2}=x\right\}$. We claim that none of the elements in $K$ has an inverse in $K$. Say, $x \tau=x^{t}, 1<t \leqq p-1$, then $x \sigma^{i}=x^{t^{2 i}}$ $=x^{\left(t^{i}\right)^{2}}, 1 \leqq i \leqq(p-1) / 2$. Since $\sigma$ is of order $(p-1) / 2,\left(t^{i}\right)^{2} \not \equiv\left(t^{j}\right)^{2} \bmod p$, $i \neq j, 1 \leqq i, j \leqq(p-1) / 2$. Hence, these $\left(t^{i}\right)^{2}, i=1,2, \cdots,(p-1) / 2$, are incongruent quadratic residues of $p$. Since $p \equiv 3 \bmod 4,-1$ is not a quadratic residue. Since the product of a quadraric residue and a quadratic nonresidue is a quadratic nonresidue, $-\left(t^{2}\right)^{i}, i=1,2, \cdots$, $(p-1) / 2$, are quadratic nonresidues. Hence, none of $x^{-\left(t^{i}\right)^{2}}, i=1,2$, $\cdots,(p-1) / 2$, is in $K$.

We form the Cayley graph, $X_{H, K}$, of $H$ with respect to $K$. Clearly, $\sigma$ is an automorphism of $X_{H, K}$. Since $H$ is abelian, by Lemma 1 the right regular representations (say, generated by $R_{x}$ ) are also autmorphisms of $X_{H, K}$. Hence, the group, $\left(\left(R_{x}, \sigma\right)\right)$, generated by $R_{x}$ and $\sigma$ is $\subseteq G\left(X_{H, K}\right)$. By Lemma $2,\left(\left(R_{x}, \sigma\right)\right)$ is not doubly transitive since $X_{H, K}$ is neither null nor complete.

We claim that $\left(\left(R_{x}, \sigma\right)\right)$ is an 2 set-transitive permutation group. For every pair $i, j=1,2, \cdots, p$ and $i \neq j$, either $\left[x^{i}, x^{j}\right] \in E\left(X_{H, K}\right)$ or $\left[x^{j}, x^{i}\right] \in E\left(X_{H, K}\right)$. If $\left[x^{i}, x^{j}\right]=\left[x^{i}, x^{i} x^{j-i}\right] \in E\left(X_{H, K}\right)$, i.e., $x \sigma^{r} \in K$ where $x^{j-i}=x \sigma^{r}$, then $x\left(\sigma^{r}\right)^{-1} \in K$, i.e., $\left[x^{j}, x^{j}\left(x\left(\sigma^{r}\right)^{-1}\right)\right]=\left[x^{j}, x^{j} x^{i-j}\right]$ $=\left[x^{j}, x^{i}\right] \in E\left(X_{H, K}\right)$. Any two edges in $X_{H, K}$ are in the form $\left[x^{i}, x^{i}\left(x \sigma^{t}\right)\right]$ and $\left[x^{j}, x^{j}\left(x \sigma^{u}\right)\right]$. We denote them by $E_{1}$ and $E_{2}$ respectively. If $t=u$, then $E_{1} R_{x}^{j-1}=E_{2}$. If $t \neq u$ then $\sigma^{u-t}$ followed by $R_{x}^{r}$, for 
some $r$, sends $E_{1}$ onto $E_{2}$. Hence, $\left(\left(R_{x}, \sigma\right)\right)$ is an 2 set-transitive permutation group and is not doubly transitive.

Corollary. Let $p$ be a prime such that $p \equiv 3 \bmod 4$. Then there exists an $p-2$ set-transitive permutation group on $\Omega_{p}$ which is not doubly transitive.

It follows from our theorem and Theorem 3 in [1].

Added in proof (June 6, 1966). After the present note was submitted, Professor R. A. Beaumont informed me that the theorem in this note was also obtained by D. Livingstone and A. Wagner in Transitivity of finite permutation groups on unordered sets, Math. Z. 90 (1965), 393-403. However, the methods are different.

\section{REFERENCES}

1. R. A. Beaumont and R. P. Peterson, Set-transitive permutation groups, Canad. J. Math. 7 (1955), 35-42.

2. H. Wielandt, Finite permutation groups, Academic Press, New York.

University of Pittsburgh 